\title{
A multi-colour study of the dark GRB 000210 host galaxy and its environment ${ }^{\star}$
}

J. Gorosabel ${ }^{1,2,3}$, L. Christensen ${ }^{4,5}$, J. Hjorth ${ }^{4}$, J. U. Fynbo ${ }^{6,4}$, H. Pedersen ${ }^{4}$, B. L. Jensen ${ }^{4}$, M. I. Andersen 5 , N. Lund ${ }^{1}$, A. O. Jaunsen ${ }^{7}$, J. M. Castro Cerón ${ }^{8}$, A. J. Castro-Tirado ${ }^{2}$, A. Fruchter ${ }^{9}$, J. Greiner ${ }^{5,10}$, E. Pian ${ }^{11}$, P. M. Vreeswijk ${ }^{7}$, I. Burud ${ }^{9}$, F. Frontera ${ }^{12,13}$, L. Kaper ${ }^{14}$, S. Klose ${ }^{15}$, C. Kouveliotou ${ }^{16}$, N. Masetti ${ }^{13}$, E. Palazzi ${ }^{13}$, J. Rhoads ${ }^{9}$, E. Rol ${ }^{14}$, I. Salamanca ${ }^{14}$, N. Tanvir ${ }^{17}$, R. A. M. J. Wijers ${ }^{14}$, and E. van den Heuvel ${ }^{14}$

${ }^{1}$ Danish Space Research Institute, Juliane Maries Vej 30, 2100 Copenhagen $\varnothing$, Denmark e-mail: nl@dsri.dk

2 Instituto de Astrofísica de Andalucía (IAA-CSIC), PO Box 03004, 18080 Granada, Spain e-mail: [jgu, ajct]@iaa.es

3 Laboratorio de Astrofísica Espacial y Física Fundamental (LAEFF-INTA), PO Box 50727, 28080, Madrid, Spain e-mail: jgu@laeff.esa.es

${ }^{4}$ Astronomical Observatory, University of Copenhagen, Juliane Maries Vej 30, 2100 Copenhagen Ø, Denmark e-mail: [lise, jens, holger, brian_j]@astro.ku.dk

5 Astrophysikalisches Institut, 14482 Potsdam, Germany e-mail: [lchristensen, mandersen, jgreiner]@aip.de

6 Department of Physics and Astronomy, University of Århus, Ny Munkegade, 8000 Århus C, Denmark e-mail: jfynbo@phys.au.dk

${ }^{7}$ European Southern Observatory, Casilla 19001, Santiago 19, Chile e-mail: [ajaunsen, pvreeswi]@eso.org

${ }^{8}$ Real Instituto y Observatorio de la Armada, Sección de Astronomía, 11.110 San Fernando-Naval (Cádiz), Spain e-mail: josemari@alumni.nd.edu

9 Space Telescope Science Institute, 3700 San Martin Drive, Baltimore, MD 21218, USA e-mail: [fruchter, burud, rhoads]@stsci.edu

10 Max-Planck-Institut für extraterrestrische Physik, 85741 Garching, Germany e-mail: jcg@mpe.mpg.de

11 Osservatorio Astronomico di Trieste, Via G.B. Tiepolo 11, 34131, Trieste, Italy e-mail: pian@tesre.bo.cnr.it

12 Dipartimento di Fisica, Università di Ferrara, Via Paradiso 12, 44100 Ferrara, Italy e-mail: frontera@fe.infn.it

13 Istituto Tecnologie e Studio Radiazioni Extraterrestri, CNR, Via Gobetti 101, 40129 Bologna, Italy e-mail: [filippo, masetti, eliana]@bo.iasf.cnr.it

14 University of Amsterdam, Kruislaan 403, 1098 SJ Amsterdam, The Netherlands e-mail: [lexk, evert, isabel, rwijers, edvdh]@science.uva.nl

15 Thüringer Landessternwarte Tautenburg, 07778 Tautenburg, Germany e-mail: klose@tls-tautenburg.de

16 NASA MSFC, SD-50, Huntsville, AL 35812, USA e-mail: kouveliotou@eagles.msfc.nasa.gov

17 Department of Physical Sciences, University of Hertfordshire, College Lane, Hatfield, Herts AL10 9AB, UK e-mail: nrt@star.herts.ac.uk

Received 13 November 2002 / Accepted 16 December 2002

Send offprint requests to: J. Gorosabel, e-mail: jgu@dsri.dk

* Based on observations collected at the European Southern Observatory, in La Silla and Paranal (Chile), ESO Large Programmes 165.H-0464(E), 165.H-0464(I) and 265.D-5742(C), granted to the GRACE Team. Based on observations made with the Danish 1.54-m telescope, in La Silla (Chile). 
Abstract. We present $U B V R I Z J \mathrm{~s} H K$ s broad band photometry of the host galaxy of the dark gamma-ray burst (GRB) of February $10,2000$. These observations represent the most exhaustive photometry given to date of any GRB host galaxy. A grid of spectral templates have been fitted to the Spectral Energy Distribution (SED) of the host. The derived photometric redshift is $z=0.842_{-0.042}^{+0.054}$, which is in excellent agreement with the spectroscopic redshift $(z=0.8463 \pm 0.0002)$ proposed by Piro et al. (2002) based on a single emission line. Furthermore, we have determined the photometric redshift of all the galaxies in an area of $6^{\prime} \times 6^{\prime}$ around the host galaxy, in order to check for their overdensity in the environment of the host. We find that the GRB 000210 host galaxy is a subluminous galaxy $\left(L \sim 0.5 \pm 0.2 L^{\star}\right)$, with no companions above our detection threshold of $0.18 \pm 0.06 L^{\star}$. Based on the restframe ultraviolet flux a star formation rate of $2.1 \pm 0.2 M_{\odot} \mathrm{yr}^{-1}$ is estimated. The best fit to the SED is obtained for a starburst template with an age of $0.181_{-0.026}^{+0.037} \mathrm{Gyr}$ and a very low extinction $\left(A_{\mathrm{V}} \sim 0\right)$. We discuss the implications of the inferred low value of $A_{\mathrm{V}}$ and the age of the dominant stellar population for the non-detection of the GRB 000210 optical afterglow.

Key words. gamma rays: bursts - galaxies: fundamental parameters - techniques: photometric

\section{Introduction}

The origin of cosmological Gamma-Ray Bursts (GRBs) remains one of the great mysteries of modern astronomy (van Paradijs et al. 2000). Over the past half decade many advances have been made in understanding the nature of the bursts and their afterglows throughout the electromagnetic spectrum. There are at present mainly two sets of models for GRBs. One set of models predicts that GRBs occur when two collapsed objects (such as black holes or neutron stars) merge (Eichler et al. 1989; Mochkovitch et al. 1993). The time-scale for binary compact objects to merge is large ( $\geqq 1 \mathrm{Gyr}$ ), so GRBs can occur after massive star formation has ended in a galaxy. The other major set of models predicts that GRBs are associated with the death of massive stars (supernovae or hypernovae) (Woosley 1993; Paczyński 1998; MacFadyen \& Woosley 1999). In this case GRBs will coincide with the epoch of star formation in the host. By determining the Spectral Energy Distribution (SED) and star formation rate $(S F R)$ of a sample of GRB host galaxies we can distinguish between these two families of GRB progenitor models (see also Belczynski et al. 2002). Substantial insight has already been gained about the galaxies that the bursts occur in. Radio, optical and/or infrared afterglows have been observed for $\sim 40 \mathrm{GRBs}$, and the majority of these coincide with starforming galaxies.

As GRB host galaxies tend to be faint $(R>23)$ spectroscopic studies of the SED are only reachable with $8-10$ m class telescopes. A cheaper and elegant alternative to spectroscopy is to extract information on the properties of the host galaxies based on multicolour broad band imaging. By determining the colours of GRB host galaxies we can derive or constrain the age of the predominant stellar population as well as the extinction. As part of the global fit, the photometric redshift of the host galaxies can be derived if the redshift is not known in advance from spectroscopic observations of the afterglow and/or the host galaxy. Additional advantages of the multicolour photometric studies compared to spectroscopic techniques are their simplicity and their multi-object feasibility. The photometric technique allows the determination of the colours of all objects in the field down to the imaging flux limit, thereby in principle permitting the study of the host galaxy environment. The precision of the photometric redshift estimate (which depends on the photometric accuracy, the spectral coverage and the number of bands) is evidently not as accurate as the spectroscopic one, but it is sufficient for a first order study of host galaxy environments.
So far it has been possible to detect optical afterglows for only about 30\% of localised GRBs (Fynbo et al. 2001; Lazzati et al. 2002). It is important to understand the nature of the remaining (rather ill-termed) so-called dark GRBs if we wish to get a complete understanding on GRB selected galaxies and thereby constrain the GRB progenitors as well as the distribution of cosmic star formation over different modes (e.g. Ramirez-Ruiz et al. 2002; Venemans \& Blain 2001). GRB 000210 is currently one of only few systems that allow a detailed study of a galaxy hosting a dark GRB. The burst exhibited the highest $\gamma$-ray peak flux among the 54 GRBs localized during the entire BeppoSAX operation, from 1996 to 2002 (Piro et al. 2002). However, no optical afterglow (OA) was detected in spite of a deep search $(R>23.5)$ carried out $\sim 16 \mathrm{hrs}$ after the gamma-ray event (Gorosabel et al. 2000a). X-ray observations performed with the Chandra X-ray telescope $21 \mathrm{hrs}$ after the GRB localised the X-ray afterglow of the burst to an accuracy of $2^{\prime \prime}$, later improved by Piro et al. (2002) to a 0 .' 6 radius error circle. The optical search revealed an extended constant source coincident with the X-ray afterglow which was proposed as the GRB host galaxy (Gorosabel et al. 2000b). In addition, Piro et al. (2002) have reported the detection of a radio transient at $8.5 \mathrm{GHz}$ spatially coincident with the X-ray afterglow. Based on the detection of a single host galaxy spectral line, interpreted to be due to [O II], Piro et al. (2002) proposed a redshift of $z=0.8463 \pm 0.0002$. Recently Berger et al. (2003) and Barnard et al. (2003) have reported $\sim 2.5 \sigma$ detections of sub-mm emission towards the position of GRB 000210 interpreted as emission from the host galaxy and hence suggesting a $S F R$ of several hundred $M_{\odot} \mathrm{yr}^{-1}$.

In this paper we present the most intensive multi-colour host galaxy imaging performed to date. The host galaxies SED studies to date had a limited number of bands (Sokolov et al. 2001; Chary et al. 2002) and no photometric redshift determinations. Throughout, the assumed cosmology will be $\Omega_{\Lambda}=0.7$, $\Omega_{M}=0.3$ and $H_{0}=65 \mathrm{~km} \mathrm{~s}^{-1} \mathrm{Mpc}^{-1}$ (except in Sect. 5.3 where the host galaxy luminosity is rescaled to the cosmology used by Lilly et al. 1995). At the proposed spectroscopic redshift $(z=0.8463)$, the look back time is $7.59 \mathrm{Gyr}(52.4 \%$ of the present age) and the luminosity distance is $1.79 \times 10^{28} \mathrm{~cm}$. The physical transverse size of one arcsec at $z=0.8463$ corresponds to $8.24 \mathrm{kpc}$.

\section{Observations and photometry}

We have used a number of optical/near-IR (NIR) resources in order to compose a well sampled SED (see Table 1). UBVI 
Table 1. Chronologically ordered optical and NIR observations carried out for the GRB 000210 host galaxy.

\begin{tabular}{lccccc}
\hline $\begin{array}{l}\text { Telescope } \\
\text { (+Instrument) }\end{array}$ & Filter & Date UT & $\begin{array}{c}T_{\text {exp }} \\
(\mathrm{s})\end{array}$ & Seeing & $\begin{array}{c}\text { Limiting magnitude } \\
(4 \sigma)\end{array}$ \\
\hline 8.2VLT (+FORS1) & $R$ & $25.237-25.240 / 10 / 00$ & 300 & 0.70 & $25.4^{\star \star}$ \\
3.58NTT (+SOFI) & $H$ & $02.251-02.410 / 09 / 01$ & $182 \times 60$ & 0.90 & 22.8 \\
3.6ESO (+EFOSC2) & $V$ & $13.219-13.253 / 09 / 01$ & $4 \times 600$ & 1.75 & 25.4 \\
3.6ESO (+EFOSC2) & $I$ & $13.256-13.278 / 09 / 01$ & $3 \times 600$ & 1.45 & 23.1 \\
3.6ESO (+EFOSC2) & $B$ & $13.280-13.302 / 09 / 01$ & $3 \times 600$ & 1.70 & 25.6 \\
3.6ESO (+EFOSC2) & $U$ & $13.304-13.348 / 09 / 01$ & $6 \times 600$ & 1.55 & 24.7 \\
8.2VLT (+ISAAC) & $K \mathrm{~s}$ & $21.159-21.193 / 09 / 01$ & $30 \times 60$ & 0.45 & 22.2 \\
8.2VLT (+ISAAC) & $J \mathrm{~s}$ & $21.194-21.218 / 09 / 01$ & $15 \times 120$ & 0.60 & $24.1^{\dagger}$ \\
8.2VLT (+ISAAC) & $J \mathrm{~S}$ & $23.193-23.218 / 09 / 01$ & $15 \times 120$ & 0.75 & $24.1^{\dagger}$ \\
1.54D (+DFOSC) & $Z$ & $19.090-19.254 / 12 / 01$ & $14 \times 600$ & 1.10 & $22.9^{\star}$ \\
1.54D (+DFOSC) & $Z$ & $20.042-20.394 / 12 / 01$ & $21 \times 600$ & 1.15 & $22.9^{\star}$ \\
\hline
\end{tabular}

$\star \star$ Published in Piro et al. (2002).

$\dagger$ The images were coadded resulting in just a single $J_{\mathrm{s}}$-band magnitude.

$\star$ The images were coadded resulting in just a single $Z$-band magnitude.

observations were carried out with the 3.6-m ESO telescope (3.6ESO) equipped with EFOSC2, covering a field of view (FOV) of $5.5 \times 5.5$. These observations were carried out in $2 \times 2$ binning mode, providing a pixel scale of $0.32 /$ pix. $R$-band measurements were obtained with the UT1 of the $8.2-\mathrm{m}$ Very Large Telescope (8.2VLT) equipped with FORS1 and are published in Piro et al. (2002). The Z-band observations were carried out during two consecutive nights with the 1.54-m Danish Telescope (1.54D) equipped with DFOSC, which provides a FOV of $13: 7 \times 13: 7$ and a pixel scale of $0 ! 39 /$ pix.

The $H$-band observations were acquired with the $3.58-\mathrm{m}$ New Technology Telescope (3.58NTT) using SOFI in the large FOV mode, which provides a FOV of $4.9 \times 4.9$ and a pixel scale of 0. '292/pix. The $J_{\mathrm{s}}$ and $K$ s-band observations are based on the UT1 of the 8.2VLT equipped with ISAAC, allowing us to cover a FOV of $2.5 \times 2$.'5 with a pixel scale of 0 ' $148 /$ pix. In Table 1 we provide the observing log of our optical and NIR observations.

Given that every extended source shows a different photodensity profile (or FWHM), an unique fixed Aperture Photometry (or static aperture photometry, AP hereafter) would yield unsatisfactory results. On the other hand, Isophotal Photometry (IP) would also not provide optimum photometry, since performing IP we would not consider the same fraction of each galaxy in the different bands due to colour-dependent morphologies and seeing. To solve this problem the total integrated photometry given by SExtractor was used (Bertin \& Arnouts 1996). For each object SExtractor performs two types of total integrated photometry: the Adaptative Aperture Photometry (AAP) and the Corrected Isophotal Photometry (CIP). The AAP and CIP supersede the values given by the AP and IP, respectively, applying to them an aperture correction. For each object SExtractor considers the photometry output given by the AAP, except if a neighbour is found biasing the flux by more than $10 \%$. If this is the case, SExtractor chooses the value given by the CIP (see Bertin \& Arnouts 1996 for details). The host galaxy of GRB 000210 is well isolated and hence its photometry is not affected by any neighbours.
The UBVRIZJs $H K$ s-band magnitudes of the host can be seen in Table 2. The UBVRI-band calibration is based on the secondary standards given in Table 2 of Piro et al. (2002). The JHKs-band calibration was performed observing the standard fields sj9105 and sj9172 (Persson et al. 1998) at several airmasses. The derived NIR secondary standards are given in Table 3 and displayed in Fig. 1. The Z-band calibration was carried out observing the spectro-photometric standard stars LTT2415 and LTT1788 (Hamuy et al. 1994) with the 1.54D at an airmass similar to that of the GRB field. The host galaxy BVRI-band magnitudes reported by Piro et al. (2002) are consistent with our magnitudes displayed in Table 2.

In order to derive the corresponding effective wavelengths and $A B$ offsets we convolved each filter transmission curve with the corresponding CCD efficiency curve (see Table 2). The $A B$ offset is defined as $A B$ off $=m_{A B}-m$, where $m$ is the magnitude in the Vega system and $m_{A B}$ is the magnitude in the $A B$ system (given by $m_{A B}=-2.5 \times \log f_{v}-48.60$, being $f_{v}$ the flux density in erg $\mathrm{s}^{-1} \mathrm{~cm}^{-2} \mathrm{~Hz}^{-1}$ ).

The $A B$ offsets of the nine bands have been derived convolving the Vega spectrum taken from the GISSEL98 (Bruzual $\&$ Charlot 1993) library ( $\alpha$ Lyrae $m=0$ in all bands by definition) with our $U B V R I Z J_{\mathrm{s}} H K$ s-band filters and the corresponding $C C D$ efficiency curves. The derived $A B$ offsets (displayed in the last column of Table 2) are similar to the ones reported by Fukugita et al. (1995).

\section{Method: Reproducing the host galaxy photometry by means of synthetic and observed SED templates}

The fit of the SEDs have been carried out using Hyperz ${ }^{1}$ (Bolzonella et al. 2000). Eight synthetic spectral types were used representing Starburst galaxies (Stb), Ellipticals (E), Lenticulars (S0), Spirals (Sa, Sb, Sc and $\mathrm{Sd}$ ) and Irregular galaxies (Im). The time evolution of the $S F R$ for all

\footnotetext{
${ }^{1}$ http://webast.ast.obs-mip.fr/hyperz/
} 
Table 2. Magnitudes of the host in the UBVRIZJs $H K$ s bands. Several characteristics of the filters are displayed: filter name (1), effective wavelength (2) and bandpass width (3). The fourth column shows the measured magnitudes (in the Vega system and not corrected from Galactic reddening). To facilitate the calculation of the $A B$ magnitudes, and consequently the flux densities for each band, the $A B$ offsets are provided in the fifth column.

\begin{tabular}{lrrrr}
\hline \hline Filter & $\begin{array}{r}\text { Effective } \\
\text { name }\end{array}$ & $\begin{array}{r}\text { Bandpass } \\
\text { width }(\AA)\end{array}$ & Magnitude & $A B$ off \\
\hline$U$ (ESO\#640) & 3718.8 & 172.9 & $23.54 \pm 0.13$ & 0.73 \\
$B$ (ESO\#639) & 4372.6 & 701.4 & $24.40 \pm 0.13$ & -0.07 \\
$V$ (ESO\#641) & 5563.9 & 856.4 & $24.22 \pm 0.08$ & 0.04 \\
$R$ (ESO R_BESSEL+36) & 6608.5 & 1300.3 & $23.46 \pm 0.10^{\dagger}$ & 0.23 \\
$I$ (ESO\#705) & 7950.2 & 844.0 & $22.49 \pm 0.12$ & 0.45 \\
$Z$ (ESO\#462) & 9477.4 & 985.1 & $22.83 \pm 0.28$ & 0.56 \\
$J$ s (ISAAC) & 12498.9 & 957.8 & $21.98 \pm 0.10$ & 0.94 \\
$H$ (SOFI) & 16519.6 & 1732.3 & $21.51 \pm 0.23$ & 1.41 \\
$K$ s (ISAAC) & 21638.0 & 1637.9 & $20.94 \pm 0.14$ & 1.87 \\
\hline
\end{tabular}

$\dagger$ Published in Piro et al. (2002).

Table 3. NIR secondary standards in the GRB 000210 field.

\begin{tabular}{lccccc}
\hline \hline Name & $\alpha_{2000}$ & $\delta_{2000}$ & $J \mathrm{~s}$ & $H$ & $K \mathrm{~s}$ \\
\hline 1 & $1: 59: 21.51$ & $-40: 39: 33.4$ & $17.94 \pm 0.03$ & $17.19 \pm 0.03$ & $17.00 \pm 0.03$ \\
2 & $1: 59: 16.72$ & $-40: 40: 20.3$ & $16.77 \pm 0.07$ & $16.51 \pm 0.03$ & $16.59 \pm 0.04$ \\
3 & $1: 59: 16.27$ & $-40: 40: 27.2$ & $18.35 \pm 0.08$ & $17.69 \pm 0.04$ & $17.46 \pm 0.05$ \\
\hline
\end{tabular}

Table 4. The table displays the parameters of the best host galaxy SED fit when several IMFs, indicated in the first column, are adopted. The rest of the columns display the inferred parameters under the assumed IMF. The second column provides the confidence of the best fit (given by $\chi^{2} /$ dof). The derived photometric redshift is displayed in the third column (and the corresponding $68 \%$ and $99 \%$ percentile errors). In the fourth and fifth columns the template family of the best fitted SED and the age of the stellar population are given. The sixth column displays the derived value of the host galaxy extinction $A_{\mathrm{V}}$. The seventh column displays the derived rest frame absolute $B$-band magnitude, $M_{B}$. The last two columns give the Luminosity of the host in units of $L^{\star}$, when the luminosity functions of Schechter (1976) and Lilly et al. (1995) are used (see Sect. 5.3 for a detailed discussion). The extinction law has been fixed to follow Calzetti et al. (2000) (the effect of the adopted extinction law is discussed in Sect. 5.2). As shown in the first two rows of the table, the resolution of our template grid is not able to make a distinction between most of the properties (Age, $A_{\mathrm{V}}, M_{B}, L / L^{\star}$ ) derived for the Sa55 and MiSc79 IMFs. The photometric redshifts derived for the three IMFs are consistent, within the $99 \%$ percentile error range, with the spectroscopic redshift. However, within the $68 \%$ precentile $(\sim 1 \sigma)$ error range, only the Sa55 and MiSc79 IMFs are consistent, Sc86 is not.

\begin{tabular}{lcccccccc}
\hline \hline IMF & $\chi^{2} /$ dof & $\begin{array}{c}\text { Photometric redshift } \\
z_{-p 68 \%, p 99 \%}^{+p 68 \%, p 9 \%}\end{array}$ & Template & Age & $A_{\mathrm{V}}$ & $M_{B}$ & $L / L^{\star}$ & $L / L^{\star}$ \\
& & & & & \\
\hline Salpeter (1955) & 1.096 & $0.842_{-0.042,0.279}^{+0.054,0.158}$ & Stb & 0.181 & 0.00 & -20.16 & 0.67 & 0.35 \\
Miller \& Scalo (1979) & 1.046 & $0.836_{-0.053,0.244}^{+0.087,0.140}$ & Stb & 0.181 & 0.00 & -20.16 & 0.67 & 0.35 \\
Scalo (1986) & 0.903 & $0.757_{-0.044,0.132}^{+0.067,0.219}$ & S0 & 1.015 & 0.00 & -19.90 & 0.52 & 0.27 \\
\hline
\end{tabular}

galaxy types is represented by an exponential model, i.e. $S F R \propto \exp (-t / \tau)$, where $\tau$ is the $S F R$ time scale. Each galaxy type has a value of $\tau$ assigned. The SFR of Stb is simulated by an exponential decay in the limit when $\tau \rightarrow 0$, in other words an instantaneous $S F R$ given by a delta function. The early type galaxy spectra (E, S0) are represented by values of $\tau$ between 1 and 2 Gyr. The Spiral galaxies (Sa, Sb, Sc and Sd) have $\tau$ values ranging from 3 to $30 \mathrm{Gyr}$. The $S F R$ of Im galaxies are represented by a constant $\operatorname{SFR}(\tau \rightarrow \infty)$.

Once the population of stars is generated following the time evolution given by the assigned SFR, the mass of the newly formed population is distributed in stars following an assumed
Initial Mass Function (IMF). Three IMFs have been considered: Miller \& Scalo (1979), Salpeter (1955), and Scalo (1986). These IMFs will be abbreviated hereafter as MiSc79, Sa55 and Sc86, respectively. In Sect. 5.1 we discuss the impact of the assumed IMFs in the determination of the photometric redshift.

The newly formed stars evolve depending on their mass and metallicity following stellar tracks. In each evolutionary stage the contribution of all the individual star spectra are added yielding an integrated galaxy SED which evolves with time. For each galaxy type the evolving SEDs can be tabulated and stored creating the so called SED libraries. Bruzual \& Charlot (1993) have derived a SED library called GISSEL98 (Galaxy 


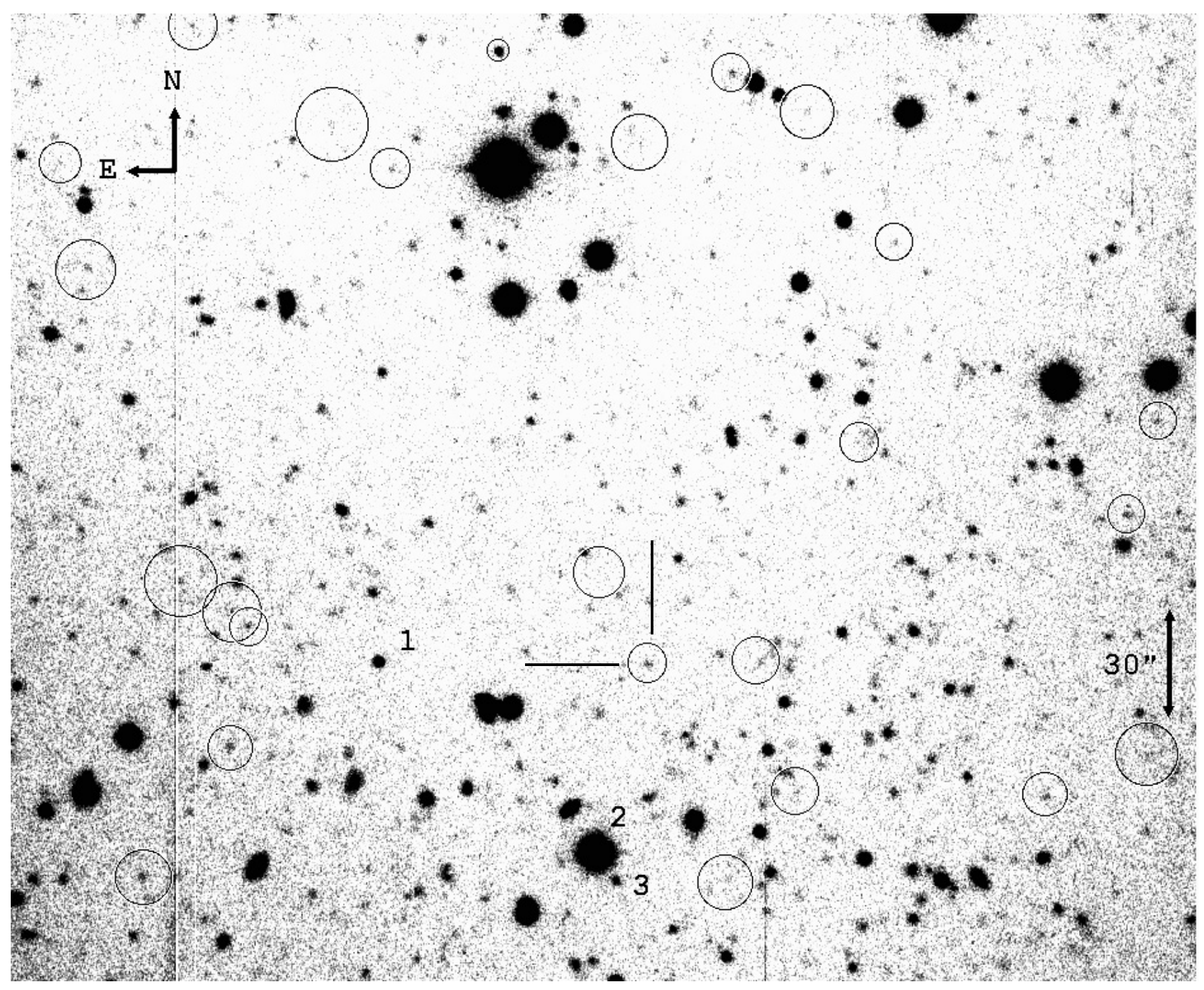

Fig. 1. The image shows the co-added $V$-band image taken at the 3.6ESO telescope at $13.219-13.253 / 09 / 01$ UT. The objects contained in the circles are the ones with redshifts consistent with $0.746<z<0.946$. As it can be seen there is no obvious concentration of these galaxies around the host. The circle radius is proportional to $1 /\left|M_{B}\right|$, so the fainter the galaxy the larger the circle. The host galaxy is indicated by the tick marks. The numbers label the secondary NIR standards shown in Table 3 . The FOV covered by the image corresponds to $5^{\prime} \times 5^{\prime}$.

Isochrone Synthesis Spectral Evolution Library), which is the base of our SED fits.

In addition to the above mentioned evolutionary templates, four averaged templates (constructed grouping the SEDs of the observed local galaxies) from Coleman et al. (1980) were considered (hereafter named as CWW). These extra spectral templates work as a backup of the evolutionary fitting SEDs, and give an approximate hint of the galaxy type when synthetic SED fits fail. The observed CWW templates can be grouped in four sets: early galaxy types (E/S0), Sbc, Scd and Im.

Furthermore, the impact of considering different extinction laws has been studied. Four extinction laws have been taken into account for the determination of the photometric redshift and the physical conditions of the GRB 000210 host galaxy, namely Calzetti et al. (2000), Seaton (1979), Fitzpatrick (1986), and Prévot et al. (1984). Each of these laws determine the dependence of the extinction on the photon frequency and are the result of different physical conditions in the interstellar space in the hosts. Thus, Seaton (1979), Fitzpatrick (1986), and Prévot et al. (1984), are appropriate to describe the Milky Way (MW), Large Magellanic Cloud (LMC) and the Small Magellanic Cloud (SMC) extinction laws, respectively. The Calzetti et al. (2000) extinction law is suitable for starburst regions. In Sect. 5.2 the effect of the adopted extinction law on the inferred host galaxy photometric redshift is discussed. In the SED fits a solar metallicity $\left(Z=Z_{\odot} \simeq 0.02\right.$, being $Z$ the mass fraction of heavy elements in the interstellar gas) have been assumed.

We varied the GRB 000210 host galaxy redshift between $z=0$ and $z=5$ with a redshift step of $\Delta z=0.01$. The host galaxy extinction was ranged in a $A_{\mathrm{V}}=0-5$ interval with a step of $\Delta A_{\mathrm{V}}=0.005 \mathrm{mag}$. Table 4 shows several inferred fit parameters for the assumed extinction law and IMFs: the fit confidence level $\left(\chi^{2} /\right.$ dof $)$, the photometric redshift $z$ (and the 


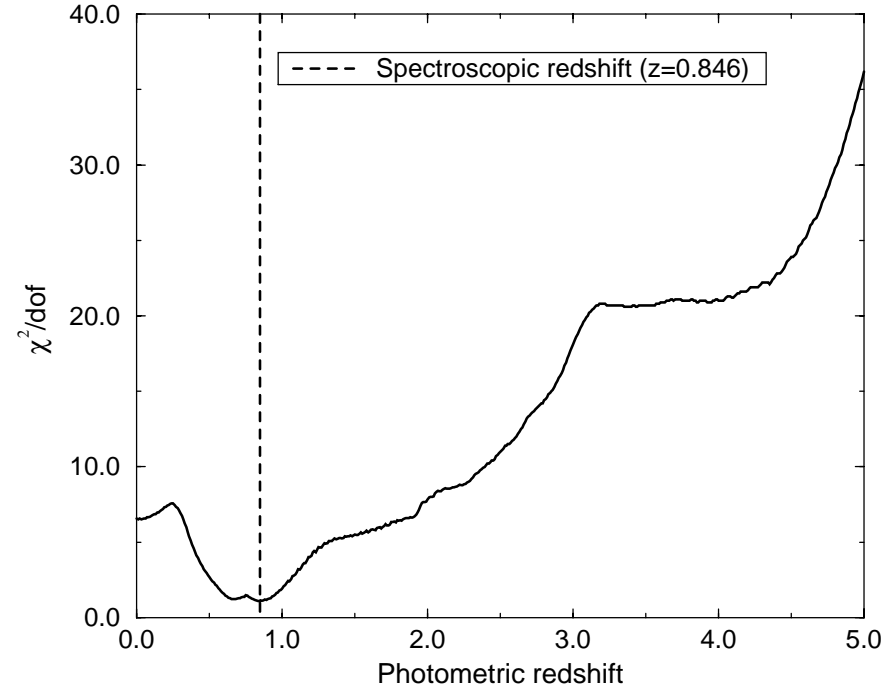

Fig. 2. The evolution of the fitted SED $\chi^{2} /$ dof as a function of the photometric redshift. The dotted vertical line indicates the spectroscopic redshift proposed by Piro et al. (2002). As shown the minimum of $\chi^{2} /$ dof (at $z=0.842$ ) is consistent with the spectroscopic redshift.

associated asymmetric uncertainties), the best fitted template, the dominant stellar age, the extinction $A_{\mathrm{V}}$, the absolute $B$-band magnitude $\left(M_{B}\right)$, and the host galaxy luminosity (in units of $\left.L^{\star}\right)$. As it is shown in Table 4 the resolution of our template grid is not able to make a distinction between most of the properties (Age, $A_{\mathrm{V}}, M_{B}, L / L^{\star}$ ) derived for the Sa55 and MiSc79 IMFs. Figure 2 shows the evolution of $\chi^{2} /$ dof as a function of the best fitted SED redshift, when a Sa55 IMF is assumed. The fit to the $U B V R I Z J_{\mathrm{s}} H K$-band photometric points shows a clear minimum around $z \sim 0.85$ and has no other acceptable redshift solutions.

\section{Study of the host galaxy environment}

At present it is unknown if GRB host galaxies preferentially are located in dense environments, or if there is any correlation between the local density of galaxies and the presence of a GRB. So far the two $z=2.04$ bursts, GRB 000301C and GRB 000926, are the only ones for which the environment of the host galaxy has been studied (Fynbo et al. 2002). In both of these fields a number of galaxies at the same redshift were detected, but the lack of blank fields studied to similar depth prevented those authors to conclude if the GRB host fields were overdense. The photometric redshifts of the galaxies in the GRB 000210 field provide the opportunity to look for other galaxies in its environment. The same calibration and photometry software used to obtain the host galaxy magnitude was applied to the rest of the objects in the field.

We consider that an object is suitable for redshift determination when it is detected at least in four bands. Objects detected in less than four filters were rejected due to the large uncertainty in the determination of their redshifts. The considered region covers a $6^{\prime} \times 6^{\prime}$ area around the host galaxy. At the redshift of the host galaxy $(z=0.8463)$ this corresponds to $\sim 3 \mathrm{Mpc} \times 3 \mathrm{Mpc}$. The SEDs used to determine the

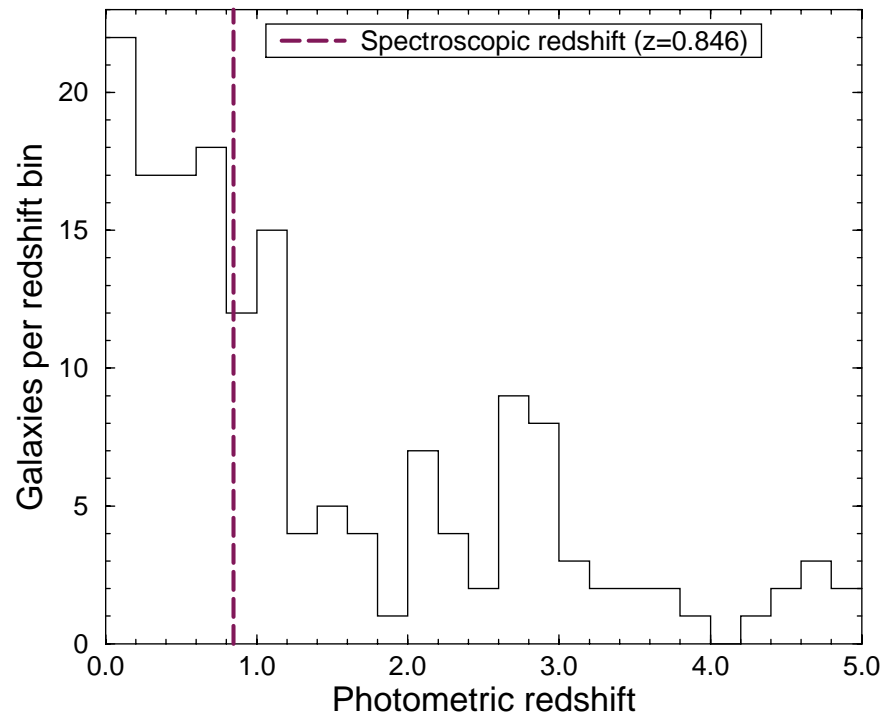

Fig. 3. The plot shows the redshift distribution of galaxies in a area of $6^{\prime} \times 6^{\prime}$ around the GRB 000210 host. The sample is made up of the 169 galaxies whose SEDs were fitted with $\chi^{2} /$ dof $<2$. The vertical dashed line shows the spectroscopic redshift. As shown there is no special concentration of galaxies with redshifts similar to the host galaxy. For the construction of the histogram we assumed a MiSc79 IMF and the extinction law given by Calzetti et al. (2000). Other IMFs and extinction laws yield similar results.

photometric redshifts of the field objects consist of 8 synthetic templates (Stb, E, S0, Sa, Sb, Sc, Sd, Im) based on a MiSc79 IMF and the extinction law given by Calzetti et al. (2000). As in the case of the host SED, four additional observed spectra from CWW were considered.

Among the 169 galaxies of the field with acceptable fits $\left(\chi^{2} /\right.$ dof $\left.<2\right)$ we considered the ones with photometric redshifts compatible (within $1 \sigma$ ) with a $\Delta z= \pm 0.1$ redshift range around the host galaxy spectroscopic redshift. In Fig. 1 a deep $V$-band image around the host galaxy is displayed. The FOV covered by the image is $5^{\prime} \times 5^{\prime}$. The circles represent the galaxies having photometric redshifts consistent with $0.7463<z<0.9463$. The radius of each circle is proportional to the inverse of the absolute $B$-band magnitude $\left(1 /\left|M_{B}\right|\right)$ of the galaxy contained inside. From the distribution of the circles on the image it is clear that there is no obvious concentration of galaxies around the host (indicated by the tick marks). The lack of clustering around the host galaxy can also be visualised in Fig. 3, where the redshift distribution of the galaxies in the field are plotted. Figure 3 shows that there is no spike of galaxies at the redshift of the host. The same study was performed considering the 200 galaxies with SEDs fitted having $\chi^{2} /$ dof $<3$, again yielding no apparent concentration of objects around the host. The exercise was repeated using several extinction laws and IMFs, giving similar results.

We have calculated the neighbour detectability threshold of our images; in other words, the minimum luminosity of a neighbour galaxy for which our data allows a photometric redshift determination. With this purpose the GRB 000210 host galaxy SED has been dimmed until detecting it only in seven bands (UBVRJs $H K s)$ above the limiting magnitudes given in 


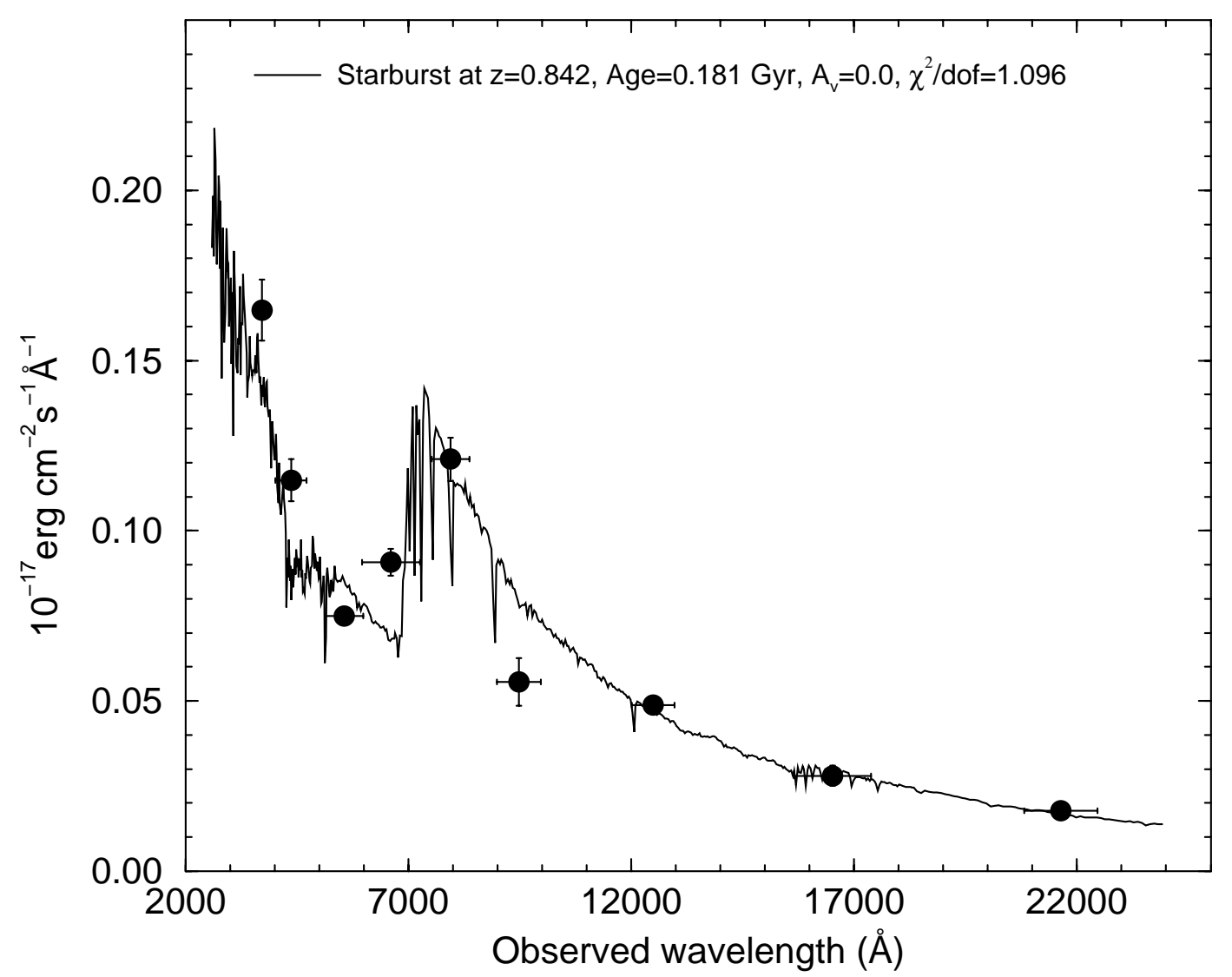

Fig. 4. The points show the measured flux in the UBVRIZJsHKs bands for the GRB 000210 host, once the Galactic dereddening is introduced (Schlegel et al. 1998). The solid curve represent the best SED fitted to the photometric points $\left(\chi^{2} / \mathrm{dof}=1.096\right)$, corresponding to a starburst synthetic template at a redshift of $z=0.842$ generated with a Sa55 IMF. The derived value of the starburst age corresponds to $0.181 \mathrm{Gyr}$. The fit is consistent with a very low extinction, $\left(A_{\mathrm{V}} \sim 0\right)$. The extinction law used to construct the plot is given by Calzetti et al. (2000). The SED

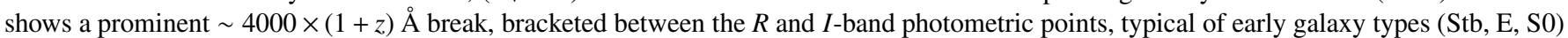
with stellar population ages $>10^{8} \mathrm{yr}$.

Table 1 . We consider that the minimum number of bands to have an acceptable redshift determination is four. So, if the host galaxy SED was dimmed in all filters by 1.16 magnitudes (see the limiting magnitudes of Table 1 and the host galaxy magnitudes of Table 2), it would have been still detected in seven bands and a secure photometric redshift determination would have been still possible. If the SED is dimmed by more than $\sim 1.29$ mag then the host would have been detected only in RJ (and may be marginally in HK), so no redshift determination would have been possible.

The absolute $B$-band magnitude of a host galaxy-like SED at $z=0.8463,1.16 \mathrm{mag}$ shallower, is $M_{B}=-19.0$. So, a photometric redshift of a neighbour galaxy (with a SED similar to the host) with $M_{B}>-19.0$ would have been indeterminable. This magnitude corresponds to a Luminosity of $L=0.23 L^{\star}$ (considering $M_{B}^{\star}=-20.6$, following Schechter 1976). The value deduced for $L$ based on Lilly et al. (1995) $\left(M_{B}^{\star}=21.33\right.$, discussed in Sect. 5.3) corresponds to $L=0.12 L^{\star}$. Thus $M_{B}=-19.0$ implies a luminosity ranging from 0.12 to $0.23 L^{\star}$ depending on the adopted $M_{B}^{\star}$ value. Therefore we consider $L=0.18 \pm 0.06 L^{\star}$ as an indication of the limiting luminosity of our host environment study. This procedure assumes that the galaxies in the host environment have similar SEDs, thus their
SEDs can be reproduced by dimming the GRB 000210 host galaxy SED by the same factor in all bands.

\section{Discussion}

\subsection{The impact of the assumed IMF on the photometric redshift}

As shown in Table 4 the spectroscopic redshift is consistent (within the $99 \%$ percentile error range) with the inferred three photometric redshifts. Thus the effect of the assumed IMF is not crucial to confirm the spectroscopic redshift of the GRB 000210 host galaxy. However, among the assumed three IMFs the MiSc79 and Sa55 IMFs are the only ones providing a photometric redshift consistent within $1 \sigma$ with the spectroscopic redshift. Thus, we consider the Sc86 IMF as the less appropriate one to describe the predominant stellar population of the GRB 000210 host galaxy.

According to Bolzonella et al. (2000) the Sa55 IMF produces an excess of bright blue stars yielding an UV flux excess. On the other hand the Sc86 IMF generates an excessive number of solar mass stars, making the spectrum too red to reproduce the observed SEDs. Intensive photometric redshift studies have 
shown that the MiSc79 IMF is a good compromise between both tendencies (Bolzonella et al. 2002).

In the particular case of the blue SED of the GRB 000210 host, the potential excess of massive stars given by the Sa55 IMF is not an inconvenient at all. The prominent UV flux predicted by $\mathrm{Sa} 55$ can easily reproduce the blue part of the observed SED. On the contrary, the excess of solar mass stars given by the Sc86 IMF is not able to reproduce the blue SED part unless the host galaxy redshift is slightly accommodated. Thus, the expected impact of the three IMFs (Bolzonella et al. 2002) is in agreement with the photometric redshifts displayed in Table 4.

\subsection{The effect of the adopted extinction law on the photometric redshift}

The host galaxy restframe SED flux density (in a $F_{\lambda}$ representation as the one of Fig. 4) increases from 3000 to $2000 \AA$ (corresponding to the observed SED between the $U$ and the $V$ band). The detection of this ionising UV continuum implies a very low extinction in the host. Given the low extinction derived for the host (see the values of $A_{\mathrm{V}}$ in Table 4) the inferred photometric redshift is basically independent of the adopted extinction law for the three IMFs. The results displayed in Table 4 for Sa55 and MiSc79 IMFs remain unchanged if the Calzetti et al. (2000) extinction law is replaced by another reddening law, as the ones given by Seaton (1979), Fitzpatrick (1986), or Prévot et al. (1984). The values of the photometric redshift derived assuming a Sc86 IMF changes slightly from $z=0.757$ to $z=0.783$, depending on the extinction law.

Therefore, in the particular case of the GRB 000210 host galaxy, the impact of the adopted extinction law on the inferred redshift is negligible and has to be considered as a second order parameter in comparison to the assumed IMF.

\subsection{Is the GRB 000210 host a subluminous galaxy?}

A subluminous galaxy is determined for having a luminosity below the knee of the luminosity function given by $L^{\star}$ (Schechter 1976). The characteristic luminosity $L^{\star}$ can be associated to a characteristic $A B$-system $B$-band absolute magnitude, $M_{B}^{\star}(A B)$, which ranges from -20.8 to -23.0 depending on the rest-frame colour of the galaxy (Lilly et al. 1995). In a more simplified approximation to the luminosity function, Schechter (1976) reports an unique value of $M_{B}^{\star}=-20.6$ (in the Vega system) for all galaxy types.

The restframe $(U-V)$ colour of the host galaxy is $(U-V)=$ -0.54 , which in the $A B$ system corresponds to $(U-V)_{A B}=$ 0.15 (see the $A B$ offsets given in Table 2). According to Table 1 of Lilly et al. (1995) this $(U-V)_{A B}$ colour implies a value of $M_{B}^{\star}(A B)=-21.40$ (given for a cosmology defined by $\Omega_{\Lambda}=$ $0, \Omega_{M}=1$ and $H_{0}=50 \mathrm{~km} \mathrm{~s}^{-1} \mathrm{Mpc}^{-1}$ ) for the redshift bin corresponding to the host. This $B$-band $A B$-system magnitude corresponds to a $B$-band absolute magnitude of $M_{B}^{\star}=-21.33$ in the Vega system (see Table 2).

The absolute $B$-band magnitude of the host galaxy for MiSc79 and Sa55 IMFs $\left(M^{\star}=-20.16\right.$ see Table 4$)$, when rescaled to the cosmology used by Lilly et al. (1995), corresponds to $M^{\star}=-20.18$. Given that $M_{B}^{\star}=-21.33$, then $L=0.35 L^{\star}$. The corresponding value of $L$ derived for a Sc86 IMF is $0.27 L^{\star}$ (see last column in Table 4).

The values of $L$, obtained using Schechter (1976), basically double (see the eighth column in Table 4) those obtained when Lilly et al (1995) is considered. Therefore, considering an averaged value of $L=0.5 \pm 0.2 L^{\star}$ for the host, we conclude that the host is very likely a subluminous galaxy. This luminosity value is consistent with the one $\left(L \approx 0.5 L^{\star}\right)$ derived by Piro et al. (2002).

\subsection{Estimation of the star formation rate}

The redshifted spectra of the GRB 000210 host galaxy have the restframe UV continuum in the observed optical range. The UV continuum emission with ongoing star formation is dominated by bright, short-lived, main-sequence $\mathrm{O}$ and $\mathrm{B}$ stars. According to Kennicutt (1998), for a Sa55 IMF (consistent with our host galaxy SED, see Table 3), the $S F R$ in a galaxy is directly proportional to the rest frame UV luminosity; $S F R_{\mathrm{UV}}=$ $1.4 \times 10^{-28} L_{v}$, where $L_{v}$ indicates the emitted energy per unit frequency around $2800 \AA$, measured in ergs s${ }^{-1} \mathrm{~Hz}^{-1}$. SFR gives the amount of stellar mass (measured in solar masses) created in the host galaxy in a restframe year. The method of deriving the $S F R$ from the UV continuum flux (named $S F R_{\mathrm{UV}}$ in the present paper) is one of several diagnostic methods used in the literature to measure $S F R \mathrm{~s}$ in galaxies (see Kennicutt 1998 for a comprehensive review). Obviously, if there is dustenshrouded star formation then this UV-based method will only provide a lower limit to the actual $S F R$.

At $z=0.8463$ the $2800 \AA$ region is redshifted to $5169.6 \AA$, so it is bracketed between the $B$ and $V$ bands. Assuming a power law SED stretch between both bands, a flux density of $0.70 \pm 0.07 \mu \mathrm{Jy}$ is estimated at $5169.6 \AA$. This flux density corresponds to a restframe $2800 \AA$ luminosity of $L_{v}=$ $1.53 \pm 0.15 \times 10^{28} \mathrm{ergs} \mathrm{s}^{-1} \mathrm{~Hz}^{-1}$, and therefore to a $S F R_{\mathrm{UV}}$ of $2.1 \pm 0.2 M_{\odot} \mathrm{yr}^{-1}$. The $S F R_{\mathrm{UV}}$ derived to date for GRB host galaxies range from 1 to $55 M_{\odot} \mathrm{yr}^{-1}$ (see Berger et al. 2003, Table 3). Thus the $S F R_{\mathrm{UV}}$ of the GRB 000210 host galaxy is in the low end of the distribution for the studied hosts. The $S F R_{\mathrm{UV}}$ per unit luminosity (considering $L \sim 0.35 L^{\star}$ based on the Sa55 IMF results of Table 4) is similar to that of other host galaxies.

As detailed in Kennicutt (1998) the above given $S F R_{\mathrm{UV}}$ estimate is more adequate for galaxies with continuous star formation (over time scales of $10^{8}$ years or longer), and provides an upper limit for younger populations such as young starburst galaxies with ages below $10^{8}$ years. For the estimated stellar population age of the host galaxy (0.181 Gyr, see Table 4), we consider that the $S F R_{\mathrm{UV}}$ expression gives still an acceptable approximation to the actual $S F R_{\mathrm{UV}}$. Kennicutt (1998) estimates that the internal uncertainty of this method is $\sim 30 \%$. This value is far from the $S F R$ derived by Berger et al. (2003) based on the tentative sub-millimeter detection of the host galaxy $\left(S F R_{\mathrm{smm}} \approx 500 M_{\odot} \mathrm{yr}^{-1}\right)$. The apparent discrepancy between $S F R_{\mathrm{smm}}$ and $S F R_{\mathrm{UV}}$ can not be explained by the internal uncertainties inherent to the $S F R_{\mathrm{Uv}}$ or $S F R_{\mathrm{smm}}$ methods. 
If the tentative detection of sub-mm emission from the host galaxy of GRB 000210 is real, as opposed to noise or emission from another source along the line of sight, we need to conclude that the host of GRB 000210 has two separate populations of massive stars. One is traced by the rest frame UV/optical light and shows no sign of extinction and the other is completely obscured by dust and is only detectable at sub$\mathrm{mm}$ wavelengths. A possible way to explain this apparently odd configuration is if the host has a clumpy and opaque ISM with no thin absorbers, which is able to completely hide part of the massive stellar population, but does not significantly affect the UV flux of the not hidden massive stars. This scenario would be consistent with the significant line of sight column density inferred from the afterglow X-ray spectrum $\left(N_{\mathrm{H}}=(5 \pm 1) \times 10^{21} \mathrm{~cm}^{-2}\right.$, Piro et al. 2002). It would also naturally explain the lack of optical afterglow emission if the progenitor was a member of the enshrouded population.

Based on the flux of the [O II] line and assuming several reasonable hypotheses Piro et al. (2002) deduced a $S F R_{[\mathrm{OII}]}$ of $\sim 3 M_{\odot} \mathrm{yr}^{-1}$. Given the impact of their assumptions (they calibrated the GRB 000210 [O II] flux relative to the one of the GRB 970828 host galaxy) and the intrinsic scatter of the $S F R_{\mathrm{UV}}$ method ( $\sim 30 \%$ according to Kennicutt 1998), we consider that our $S F R_{\mathrm{Uv}}$ estimate is in agreement with the $S F R_{[\mathrm{OII}}$ determined by Piro et al. (2002). Thus, the [O II] line and the UV continuum originate from the same unextincted blue stellar component.

\subsection{Implications of the fitted SED on the GRB progenitor}

The fitted SED assuming a MiSc79 or a Sa55 IMF is consistent with a Stb, independently of the extinction law used. The Stb template is characterized by a value of $\tau \rightarrow 0$, so the $S F R$ can be expressed by a delta function. In this scenario, the star formation is instantaneous, and occurs at the same time for all the stars, independently of their masses. Thus all the stars should have the same age. The local birth places in a host galaxy (even in the same star forming region) show different physical conditions and, besides, they would be causally separated from each other, so an instantaneous star formation is physically inviable. Therefore, this description should be considered only as an idealisation of a quasi-simultaneous starburst episode occurred around $0.181 \mathrm{Gyr}$ ago (measured in the restframe) in the host galaxy.

Several alternatives are possible to explain a GRB progenitor with an age of $\sim 0.181 \mathrm{Gyr}$. The first alternative would be a progenitor made up of a binary merging system. The life time of such systems is $\sim 0.1-1 \mathrm{Gyr}$, i.e. compatible with the host galaxy dominant stellar age (Eichler et al. 1989). Thus, the $\gamma$-bright (but optically dark) GRB 000210 would come from the collapse of a compact binary system. This interpretation would support a connection between dark GRBs and binary merging systems, which would not necessarily invoke a circumburst dense region and an extinction mechanism to explain the lack of optical emission (Castro-Tirado et al. 2002). However, a binary merging origin shows several problems.
Piro et al. (2002) derived a column density of $N_{\mathrm{H}}=(5 \pm 1) \times$ $10^{21} \mathrm{~cm}^{-2}$ along the line of sight to the burst. It is not obvious to conceive such binary systems located within a high density $\left(N_{\mathrm{H}}>10^{21} \mathrm{~cm}^{-2}\right)$ region. Each of the components of such systems is the result of an asymmetric collapse of stellar cores, providing in the instant of the explosion kick off velocities up to $900 \mathrm{~km} \mathrm{~s}^{-1}$ to the newly formed compact object (Frail et al. 1994; Nazin \& Postnov 1997). Thus the binary systems tend to be located far from their birth places, as they have $0.1-1 \mathrm{Gyr}$ to travel before the binary collapse episode occurs. However, Belczynski et al. (2002) have recently shown that, although far from the star forming regions, the binary systems should occur inside the host galaxies. Besides they find that such systems are more numerous than previously thought.

In principle a collapsar with an age of $\sim 0.181 \mathrm{Gyr}$ is not easy to accommodate. The age of a $8 M_{\odot}$ star when it explodes as a type II SN is $\sim 0.05 \mathrm{Gyr}$ (see for instance Portinari et al. 1998). More massive stars, as the progenitors suggested in the collapsar scenario (Paczyński 1998; MacFadyen \& Woosley 1999), have even shorter lifetimes.

The clumpy ISM scenario would be able to reconcile the difference between the age derived from the SED $(\sim 0.181 \mathrm{Gyr})$ and the age expected for a collapsar (the lifetime of a $\sim 100 M_{\odot}$ progenitor is $\sim 0.003 \mathrm{Gyr}$, according to Portinari et al. 1998). In such scenario the hidden population of young stars would be able to generate a collapsar but not contribute to the host galaxy SED.

\section{Conclusions}

We have presented an intensive UBVRIZJs $H K$ s broad band photometry of the GRB 000210 host galaxy which has allowed us to determine its photometric redshift. The derived photometric redshift is $z=0.842_{-0.042}^{+0.054}$, in excellent agreement with the spectroscopic redshift $(z=0.8463 \pm 0.0002)$ proposed by Piro et al. (2002). The inferred redshift is basically independent of the extinction law and IMF assumed, although (at least in the particular case of GRB 000210) the Scalo (1986) IMF provides slightly worse results than Miller \& Scalo (1979) and Salpeter (1955) IMFs. The SED of the host galaxy is consistent with a starburst template with an age of $\sim 0.181 \mathrm{Gyr}$ and a very low extinction $\left(A_{\mathrm{V}} \sim 0\right)$. Based on the restframe UV flux a star formation rate of $2.1 \pm 0.2 M_{\odot} \mathrm{yr}^{-1}$ is estimated.

The absolute restframe $B$-band magnitude of the host $\left(M_{B}=-20.16\right)$ is consistent with the distribution of the $M_{B}$ host galaxy values measured to date (see Djorgovski et al. 2001, Fig. 2). We determine a value of $L=0.5 \pm 0.2 L^{\star}$ for the luminosity of the host, in agreement with the value estimated by Piro et al. (2002).

We have tried to the explore the role played by galactic interactions triggering the GRB phenomena. Many host galaxies observed to date appear as part of complex and interacting systems (GRB 980613, Hjorth et al. 2002; GRB 001007, Castro Cerón et al. 2002). According to our study the GRB 000210 host galaxy is a subluminous galaxy with no interacting companions above $0.18 \pm 0.06 L^{\star}$.

The low value of the extinction obtained in the SED fit $\left(A_{\mathrm{V}} \sim 0\right)$ makes difficult to explain the optical darkness of 
GRB 000210 in terms of the global host galaxy dust extinction. If dust extinction is the reason of the lack of optical afterglow emission, then the circumburst region has to be very compact and localised around the progenitor. This hypothesis would agree with observations carried out for the opticallyfaint GRB 990705. Andersen et al. (2002) have localised the optically dim GRB 990705 (but NIR bright, see Masetti et al. 2000) in a face-on spiral galaxy. Thus given the thin disk of a spiral galaxy ( 0.3 kpc), the optical extinction of GRB 990705 can not be attributed to the global ISM in its host. This clumpy and fragmented ISM would also explain the apparent discrepancy between our SFR estimate (derived from the galaxy UV flux) and the one recently reported based on the sub-millimeter range (Berger et al. 2003; Barnard et al. 2003).

Several progenitor models have been discussed in order to explain the inferred stellar population age and the low host galaxy extinction. Both the collapsar and the binary merging models show severe limitations to explain the visible stellar age and the line of sight $\mathrm{H} \mathrm{I}$ column density (derived from the afterglow X-ray spectrum) respectively. A solution to this problem would be the existence of a younger population of stars (several Myr of age) hidden by the clumpy ISM. Such population (which would include the progenitor massive star) would not have any impact in the host galaxy SED. Morphological information derived by HST could verify the proposed ISM clumpy scenario present in the host galaxy of the dark GRB 000210.

Acknowledgements. J. Gorosabel acknowledges the receipt of a Marie Curie Research Grant from the European Commission. This work was supported by the Danish Natural Science Research Council (SNF). J.M. Castro Cerón acknowledges the receipt of a FPI doctoral fellowship from Spain's Ministerio de Ciencia y Tecnología. J.U. Fynbo acknowledges support from the Carlsberg Foundation. We thank our referee L. Piro for fruitful comments. The observations presented in this paper were obtained under the ESO Large Programmes $165 . \mathrm{H}-$ 0464(E), 165.H-0464(I) and 265.D-5742(C).

\section{References}

Andersen, M. I., Hjorth, J., Gorosabel, J., et al. 2002, in preparation Barnard, V. E., Blain, A. W., Tanvir, N. R., et al. 2003, MNRAS, 338, 1

Belczynski, K., Bulik, T., \& Kalogera, V. 2002, ApJ, 571, L147

Berger, E., Cowie, L. L., Kulkarni, S. R., et al. 2003, ApJ, in press [astro-ph/0210645]

Bertin, E., \& Arnouts, S. 1996, A\&AS, 117, 393

Bolzonella, M., Miralles, J.-M., \& Pelló, R. 2000, A\&A, 363, 476

Bolzonella, M., Pelló, R., \& Miralles, J.-M. 2002, posted at http://webast.ast.obs-mip.fr/hyperz/manual.html

Bruzual, A. G., \& Charlot, S. 1993, ApJ, 405, 538
Calzetti, D., Armus, L., Bohlin, R. C., et al. 2000, ApJ, 533, 682

Castro Cerón, J. M., Castro-Tirado, A. J., Gorosabel, J., et al. 2002, A\&A, 339, 445

Castro-Tirado, A. J., Castro Cerón, J. M., Gorosabel, J., et al. 2002, A\&A, 393, L55

Chary, R., Becklin, E. E., \& Armus, L. 2002, ApJ, 566, 229

Coleman, G. D., Wu, C. C., \& Weedman, D. W. 1980, ApJS, 43, 393

Djorgovski, S. G., Kulkarni, S. R., Bloom, J. S., et al. 2001, GammaRay Bursts in the Afterglow Era, ed. E. Costa, F. Frontera, \& J. Hjorth, 218

Eichler, D., Livio, M., Piran, T., \& Schramm, D. N. 1989, Nature, 340, 126

Fitzpatrick, E. L. 1986, AJ, 92, 1068

Frail, D. A., Goss, W. M., \& Whiteoak, J. B. Z. 1994, ApJ, 437, 781

Fynbo, J. P. U., Jensen, B. L., Gorosabel, J., et al. 2001, A\&A, 369, 373

Fynbo, J. P. U., Møller, P., Thomsen, B., et al. 2002, A\&A, 388, 425

Fukugita, M., Shimasaku, K., \& Ichikawa, T. 1995, PASP, 107, 945

Gorosabel, J., Jensen, B. L., Olsen, L. F., et al. 2000a, GCN, 545

Gorosabel, J., Hjorth, J., Jensen, B. L., et al. 2000b, GCN, 783

Hamuy, M., Suntzeff, N. B., Heathcote, S. R., et al. 1994, PASP, 106, 566

Hjorth, J., Thomsen, B., Nielsen, S. R., et al. 2002, ApJ, 576, 11

Kennicutt, R. C. 1998, ARA\&A, 36, 189

Lazzati, D., Covino, S., Ghisellini, G., et al. 2002, MNRAS, 330, 583

Lilly, S. J., Tresse, L., Hammer, F., Crampton, D., \& Le Févre, O. 1995, ApJ, 455, 108

MacFadyen, A. I., \& Woosley, S. E. 1999, ApJ, 524, 262

Masetti, N., Palazzi, E., Pian, E., et al. 2000, A\&A, 354, 473

Mochkovitch, R., Hernanz, M., Isern, J., \& Martin, X. 1993, Nature, 361,236

Miller, G. E., \& Scalo, J. M. 1979, ApJS, 41, 513

Nazin, S. N., \& Postnov, K. A. 1997, A\&A, 317, L79

Paczyński, B. 1998, ApJ, 494, L45

Persson, S. E., Murphy, D. C., Krzeminski, W., Roth, M., \& Rieke, M. J. 1998, AJ, 116, 2475

Piro, L., Frail, D. A., Gorosabel, J., et al. 2002, ApJ, 577, 680

Portinari, L., Chiosi, C., \& Bressan, A. 1998, A\&A, 334, 505

Prévot, M. L., Lequeux, J., Prévot, L., Maurice, E., \& Rocca-Volmerange, B. 1984, A\&A, 132, 389

Ramirez-Ruiz, E., Lazzati, D., \& Blain, A. W. 2002, ApJ, 565, L9

Salpeter, E. E. 1955, ApJ, 121, 161

Scalo, J. M. 1986, Fundam. Cosmic Phys. 11, 1

Schechter, P. 1976, ApJ, 203, 297

Schlegel, D. J., Finkbeiner, D. P., \& Davis, M. 1998, ApJ, 500, 525

Seaton, M. J. 1979, MNRAS, 187, 73

Sokolov, V. V., Fatkhullin, T. A., Castro-Tirado, A. J., et al. 2001, ApJ, 372,438

van Paradijs, J., Kouveliotou, C., \& Wijers, R. A. M. J. 2000, ARA\&A, 38, 379

Venemans, B. P., \& Blain, A. W. 2001, MNRAS, 325, 1477

Woosley, S. E. 1993, ApJ, 405, 273 